\title{
Systematical Development of NVH Engineering for Vehicle Electrical Powertrain Based on an Optimized V-Model
}

\author{
Shaoyou Shi*, Kaiyu Yu, Zecheng Zhong \\ Shanghai Electric Group Co., Ltd. Central Academe, Shanghai, China \\ Email: *shishy2@shanghai-electric.com, yuky2@shanghai-electric.com, zhongzch@shanghai-electric.com
}

How to cite this paper: Shi, S.Y., Yu, K.Y. and Zhong, Z.C. (2021) Systematical Development of NVH Engineering for Vehicle Electrical Powertrain Based on an Optimized V-Model. World Journal of Mechanics, 11, 7-15.

https://doi.org/10.4236/wjm.2021.112002

Received: June 4, 2020

Accepted: February 23, 2021

Published: February 26, 2021

Copyright $\odot 2021$ by author(s) and Scientific Research Publishing Inc. This work is licensed under the Creative Commons Attribution International License (CC BY 4.0).

http://creativecommons.org/licenses/by/4.0/

\begin{abstract}
For systematical NVH development of vehicle (especially for mass-production passenger vehicles) electric powertrain, an optimized V-Model is designed and has been implemented in the entire component-vehicle development, which integrates three individual branches: simulation, validation and optimization. Compared to the V-models in the traditional sense, this optimized $\mathrm{V}$-model is not only driven by requirement and task accomplishment but also maximum optimization of NVH system performance. In this case, developing procedures are capable to be efficiently iterated and the $\mathrm{NVH}$ engineering can be expanded into 3D with this V-model.
\end{abstract}

\section{Keywords}

V-Model, NVH (Nosie Vibration and Harshness), SEP (System Engineering Process), Electrical Powertrain, MBS (Multi-Body System), Acoustic, Electromagnetic, Gear Mesh, Topology Optimization

\section{Introduction}

The V-model concept was designed independently in Germany and in the United States in the 1980s. In research and development, it was applied for the development of advanced automation system (AAS) programs at Hughes Aircraft, which eventually formed the test strategy and showed integration approach driven by new challenges. The V-model is also used to regulate the software development process with the German federal administration and plays as standard for developers within the region. In recent years, it has been widespread applied as a vaguer illustrative depiction in research, development, production control not only in the software development but also in a variety of system engineering 
processes (SEP).

Noise, vibration and harshness ( $\mathrm{NVH}$ ) is the engineering of objective measurement, reflecting human subjective impressions analysis and performance development for component and vehicle. Its theoretical basis is multi-body dynamic and acoustic. Compared to the combustion engine vehicles, in the electrical vehicles, the internal noise source is mainly due to electromagnetic force, gear mesh force and IGBT frequency switching. Because of different structure, electrical powertrain shows a different transfer path, which is also reflected in the resonance distribution.

So, the NVH performance in electrical powertrain is a complex electromechanical transient process, including electrical current invert process from inverter, electromagnetic effect from electric motor, transmission from the gear mesh and finally becomes the noise and vibration response through the transfer path of electrical powertrain and vehicle structure to passengers, which makes the NVH development and research a product life cycle system engineering process.

In order to systematically develop and continuously improve the NVH performance, an optimized V-model is introduced in this paper including the follows: the framework of the optimized V-model is introduced in Section 2. Its branches such as simulation, validation and optimization will be introduced in detail in Sections 3, 4 and 5. The last section is the conclusion of this optimized V-model.

\section{Optimized V-Model}

Consider the original V-model from Forsberg et al. (1990) [1] in Figure 1, where the system is decomposed and defined in each level on the one hand, and then verified on the other hand to accomplish the entire SEP.

Optimized V-model has introduced the optimization process for V-model and promotes it from 2D to a 3D development model. For NVH system engineering, it is described as an optimized V-model in Figure 2.

Different from general system development, the NVH system of electrical powertrain is a progress from fuzzy to concrete state. As the development advances, the constraints, requirements and integration are constantly changing.

This V-model is capable to meet complex changing progress circumstance, integrate the developing procedures such as simulation, validation and optimization and be implantable in entire vehicle research and development in Figure 3.

\section{Simulation}

This $160 \mathrm{~kW}$ electrical powertrain for passenger vehicle includes a power electric unit (PEU), electrical motor (EM) and gearbox (GB), which is a multi-body system, and it follows the kinematic formula

$$
\overline{\overline{M(x)}} \ddot{x}-Q_{v}+C_{x}^{T} \lambda=F
$$




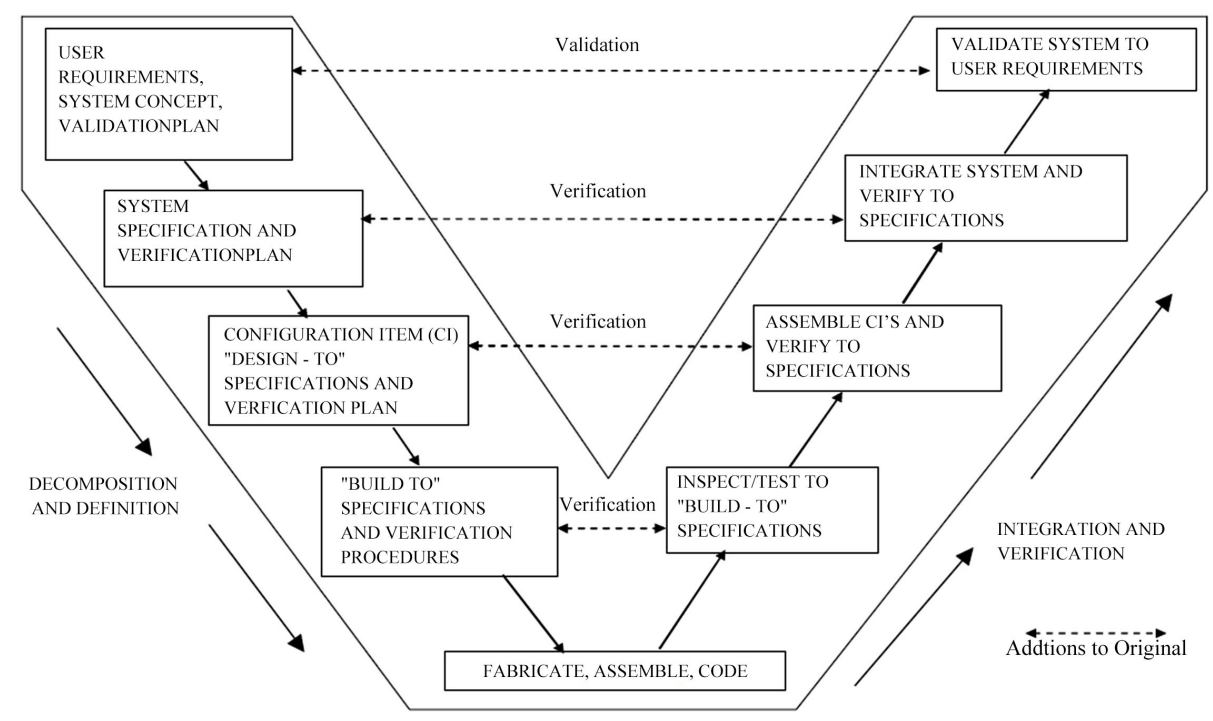

Figure 1. Original V-model.

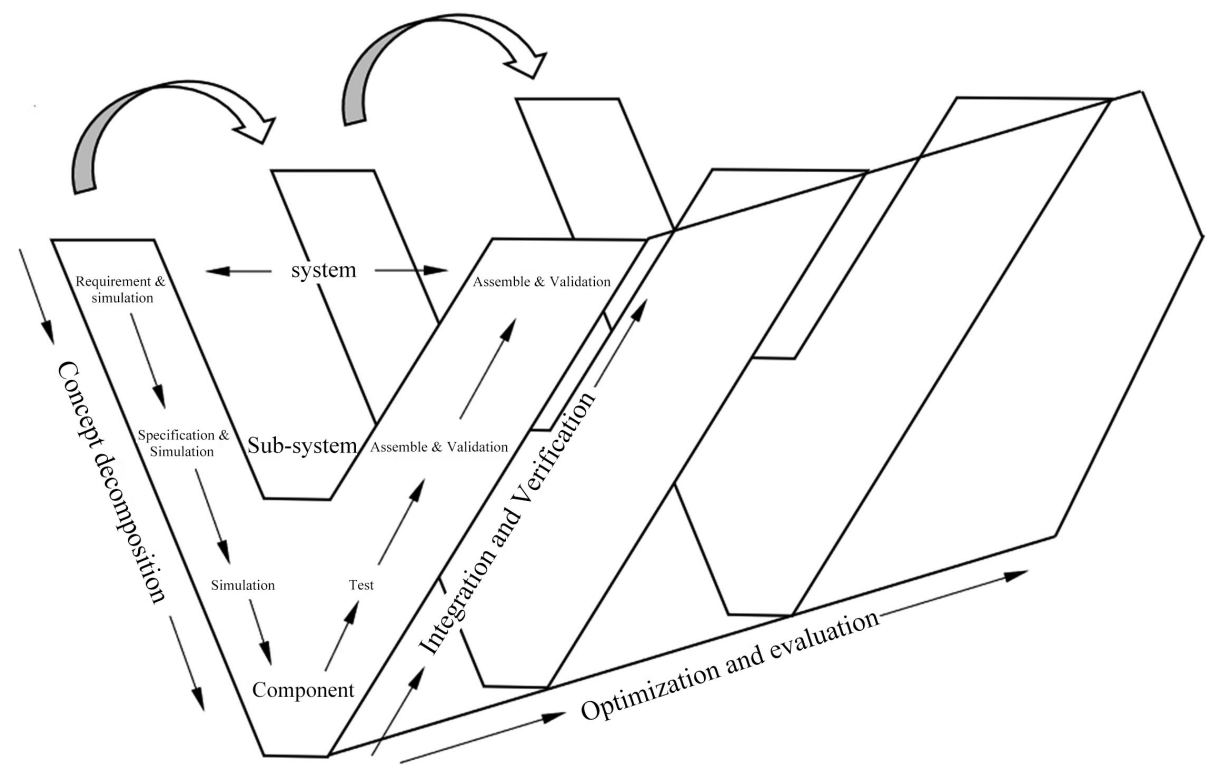

Figure 2. An optimized V-model.

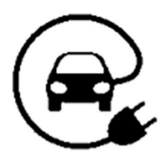

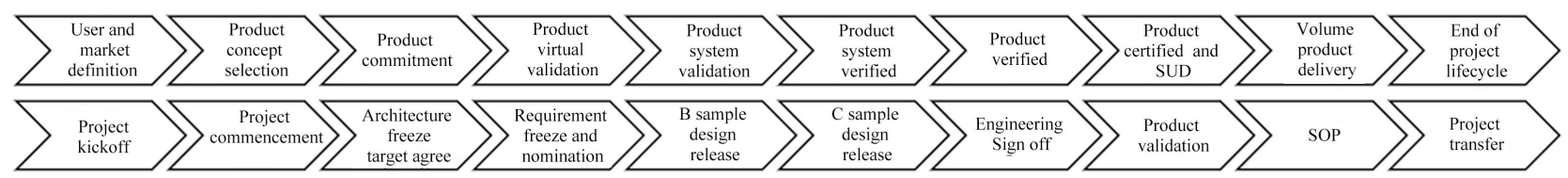

0

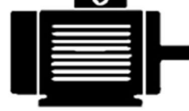

Figure 3. Electrical powertrain and vehicle research and development milestones. 


$$
C(x, \dot{x})=0
$$

where, $M(x)$ is the mass matrix, $C_{q}$ is the constraint conditions with respect to the coordinates, $\lambda$ is the lagrange multipliers and $Q_{v}$ is the quadratic velocity vector.

For MBS modelling in the computational aspects from Schielen (1991) [2] of the electrical powertrain, a rigid-flexible bodies coupling system was built in the Simcenter 3D Motion environment in Figure 4.

The shafts and gears are built as rigid bodies, while the housing is built as elastic flexible bodies with finite elements. The stiffness and damping models are applied for bearing connections. So the transient process, that gear mesh forces excite the response of system vibration, has been calculated and employed for the acoustic simulation.

On the other hand, the electromagnetic forces, which come from the magnetic field between rotor and stator, are the excitation of electromagnetic noise.

$$
F=\frac{1}{2 \mu_{0}} \oint\left(B_{n}^{2}-B_{t}^{2}\right) d l
$$

where $\mu_{0}$ stands for the magnetic permeability, $B_{n}$ is the radial magnetic flux density and $B_{t}$ is the tangential magnetic flux density. This excitation is loaded on the structure model of electrical powertrain and applied for response calculation by mode superposition method after Gieras et al. (2006) [3] in Figure 5.

The gear mesh and electromagnetic forces excite the response of electrical powertrain system vibration. And housing surface vibration leads to the surrounding vibration, thus making sound waves in Figure 6.

\section{Validation}

The NVH performance of an $160 \mathrm{~kW}$ electrical powertrain from Shanghai Electric Group Co., Ltd. has been validated in the semi-anechoic room of CATARC in Figure 7.

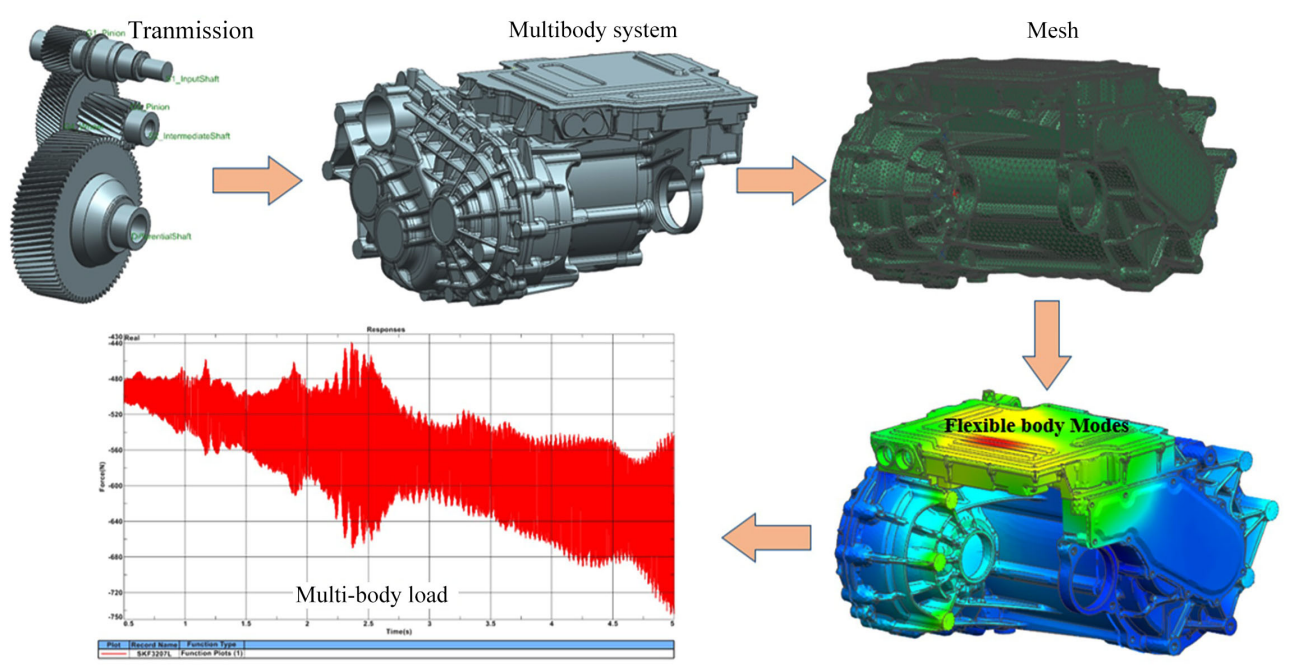

Figure 4. MBS of electrical powertrain simulation. 


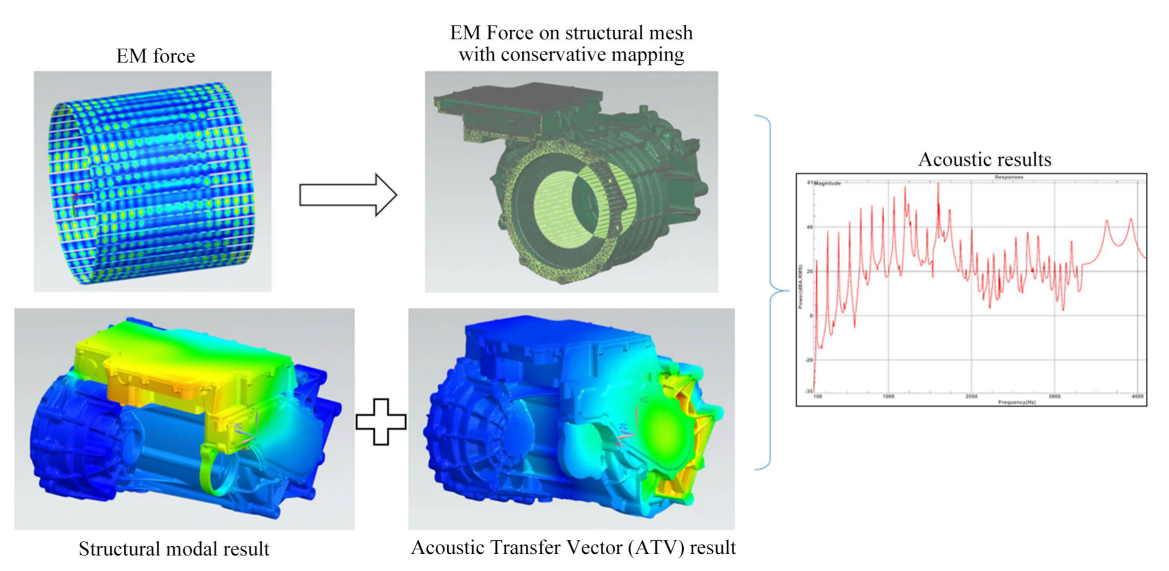

Figure 5. Electromagnetic excitation on the electrical powertrain.

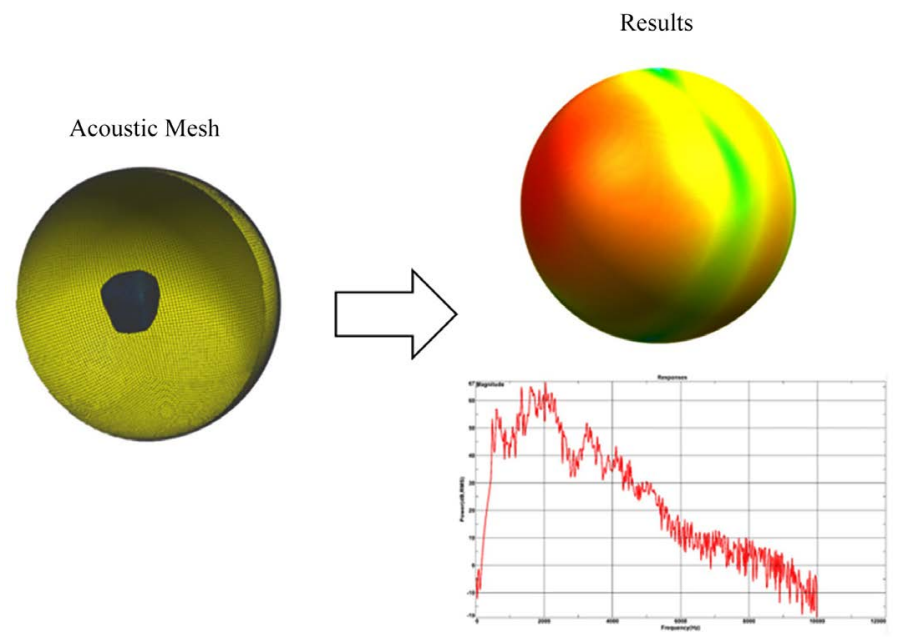

Figure 6. Acoustic simulation.

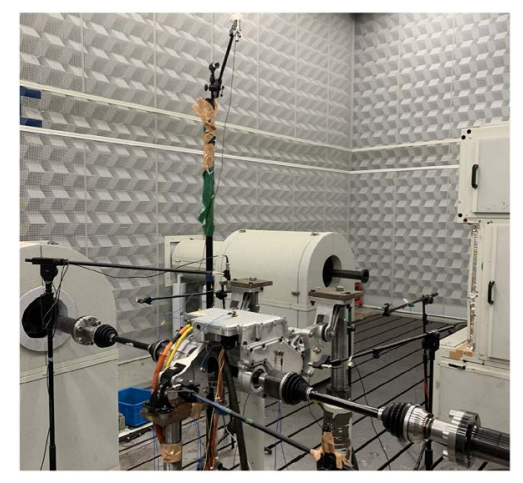

Figure 7. An $160 \mathrm{~kW}$ electrical powertrain in semi-anchoic room.

Figure 8 shows that noise from electrical powertrain is mainly contributed by IGBT switching frequency noise from PEU, electromagnetic noise from EM and gear mesh noise from GB. From the order analysis, we noticed that IGBT switching frequency noise $\left( \pm 12^{\text {th }}\right.$ order offset $\left.10 \mathrm{kHz}\right)$ appears around its PWM frequency and distributes like an umbrella. It's related to the carrier frequency and modulated frequency and happens because the DC-AC inverter brings har- 
monic current into alternating current (AC), which drives the electrical motor. From Figure 9, we noticed that it doesn't change much from mid- to high-speed range.

The electromagnetic noise $\left(24^{\text {th }}\right.$ and $48^{\text {th }}$ order), see Figure 10 , appears because of electromagnetic force. Both order noises vary themselves around 10 $\mathrm{dB}(\mathrm{A})$ in the speed range, however, the speed variations from low to high range affects the sound pressure not much compared to the torque.

Figure 11 shows that the gear mesh noise $\left(7.75^{\text {th }}\right.$ and $31^{\text {th }}$ order $)$ appears due to the gear mesh stiffness variation. From the low- to high-speed range, the gear mesh noise especially $7.75^{\text {th }}$ order shows an upward tendency.

\section{Optimization}

In the research and development progress of $\mathrm{NVH}$ engineering, optimization drives the progress of V-model forward. During the concept developing phase, the electrical powertrain system is optimized with the objective of NVH performance mainly under design constraints. In the next phase from design verification to mass-production, the NVH optimization serves the objective of consistency under production constraints. In the final phase of product delivery, the electrical powertrain is optimized with the objective of customer positive feedback.

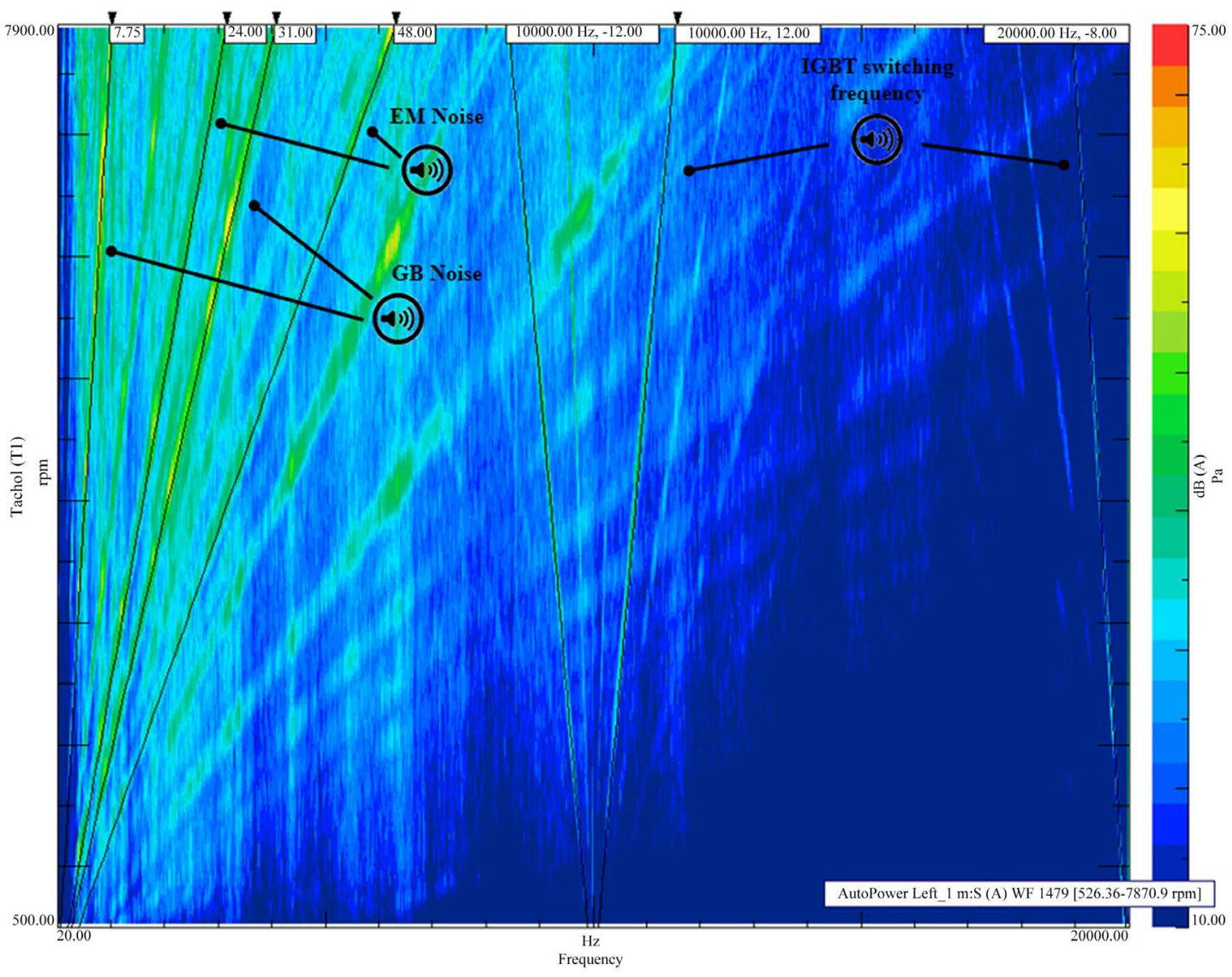

Figure 8. Characterization of electrical powertrain noise. 


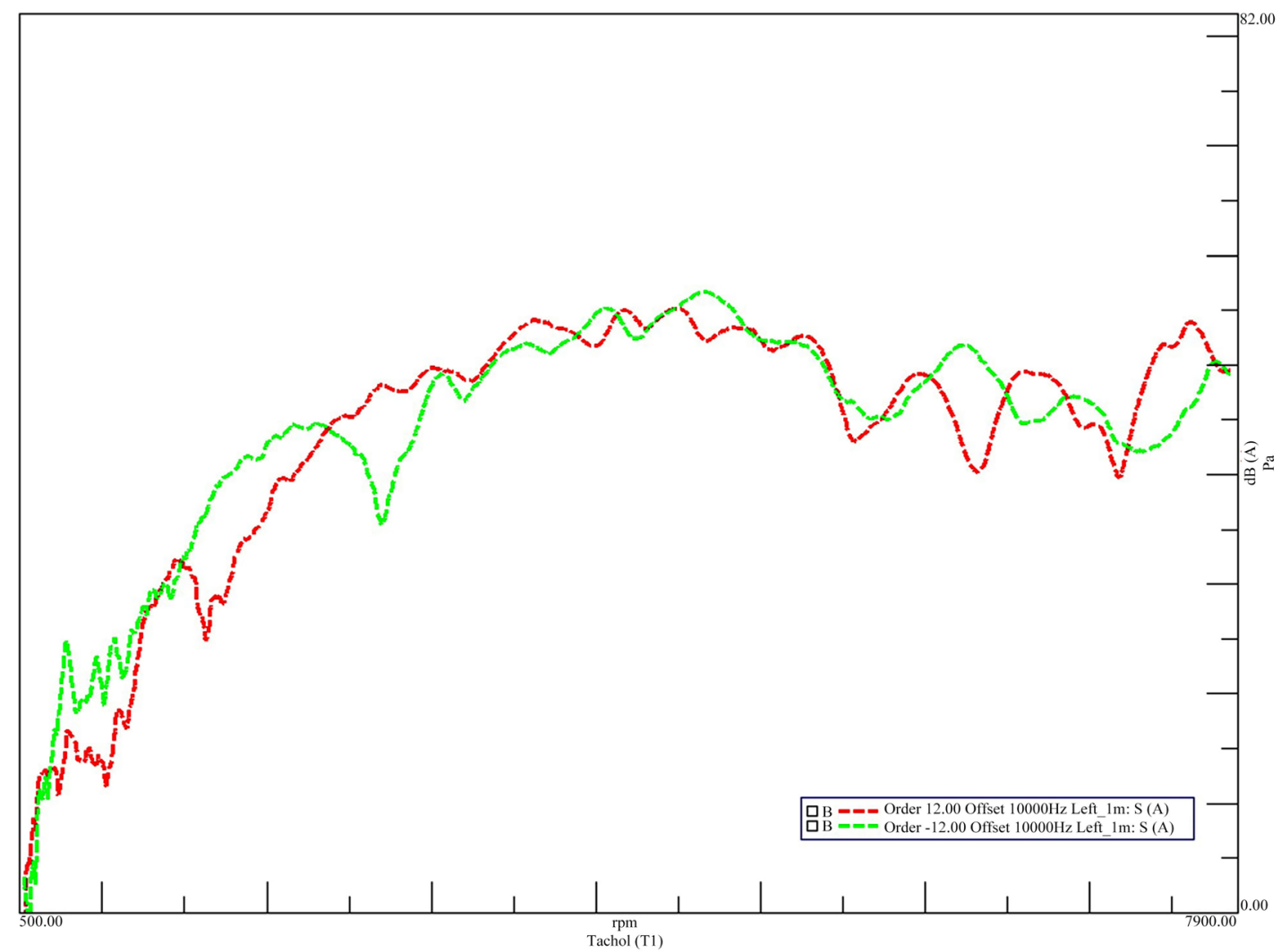

Figure 9. IGBT switching frequency noise.

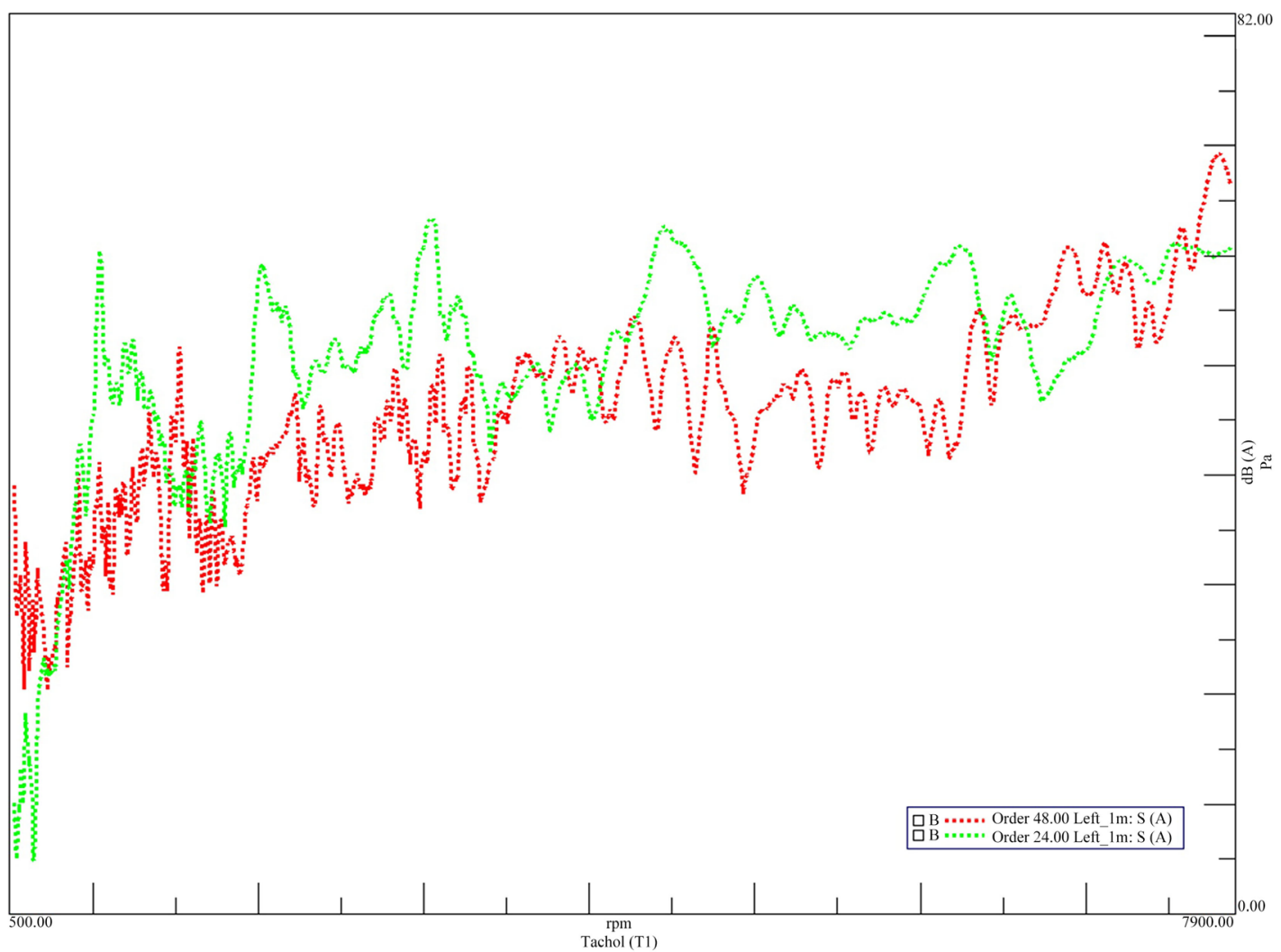

Figure 10. Electromagnetic noise. 


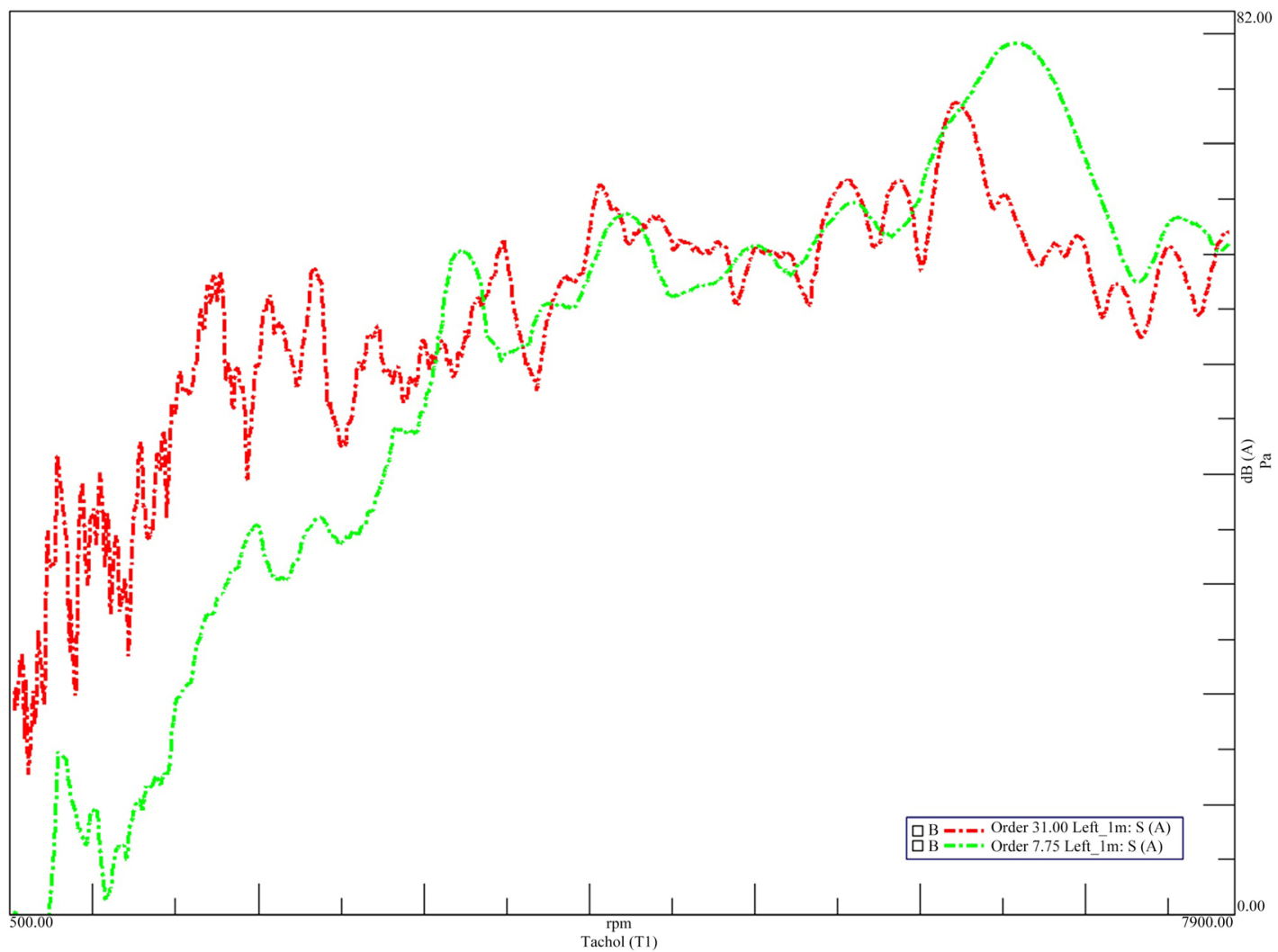

Figure 11. Gear mesh noise.

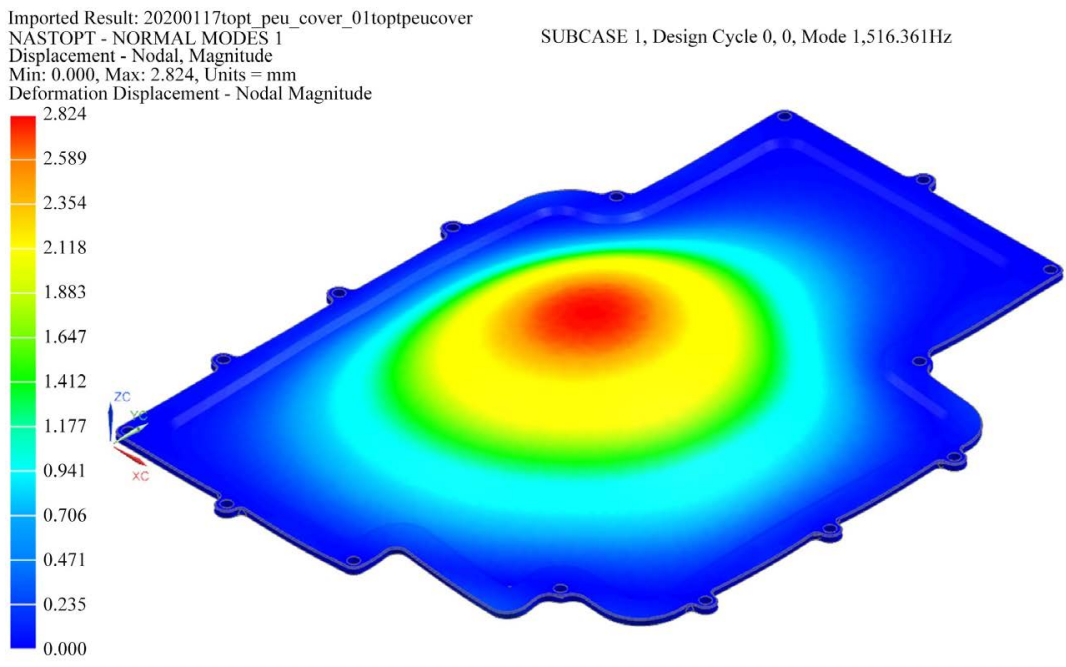

$[\mathrm{mm}]$

Figure 12. A flat part with $516 \mathrm{~Hz}$ eigenfrequency.

From our current development, with the aid of decomposition from V-model, several flat parts are considered with a low NVH performance. The following part has a relative low $1^{\text {st }}$ eigenfrequency of $516 \mathrm{~Hz}$ in Figure 12.

To improve the NVH performance, a topology optimization has been applied in the system in Figure 13. With remaining the same mass quality, the NVH performance of the part has been increased around $35.9 \%$. 

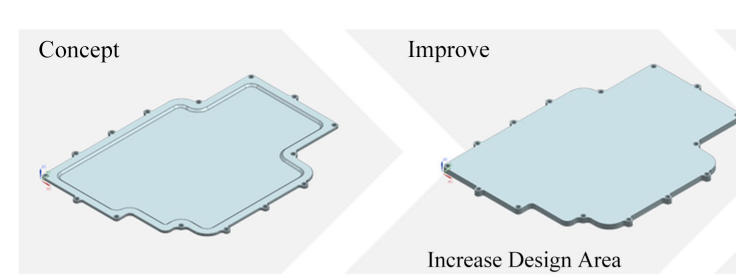

Increase Design Area

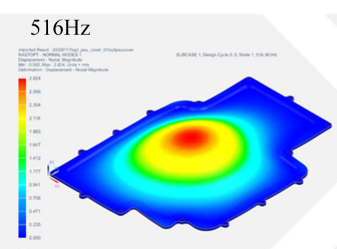

$845 \mathrm{~Hz}$

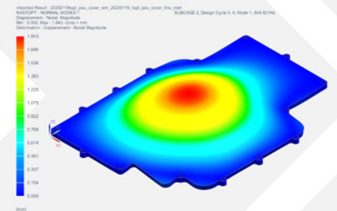

Optimization

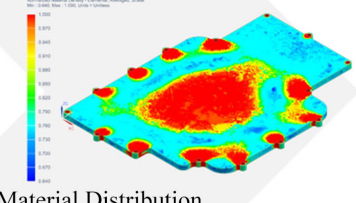

Material Distribution

$806 \mathrm{~Hz}$

Figure 13. Flat part optimization.

\section{Conclusion}

This paper presents a new V-model, which is developed for the NVH engineering of electrical powertrain research and development. This optimized V-model is capable to efficiently iterate the developing procedures and expand the development from $2 \mathrm{D}$ to $3 \mathrm{D}$ model. Three branches such as simulation, validation and optimization are introduced with examples.

\section{Conflicts of Interest}

The author declares no conflicts of interest regarding the publication of this paper.

\section{References}

[1] Forsberg, K. and Mooz, H. (1990) Proceedings of the First Annual NCOSE Conference,

[2] Schielen, W. (1991) Computational Aspects in Multibody System Dynamics. Computer Methods in Applied Mechanics and Engineering, 90, 569-582. https://doi.org/10.1016/0045-7825(91)90172-3

[3] Gieras, J.F., Wang, C. and Lai, J.C.S. (2006) Noise of Polyphase Electrical Motors. Taylor \& Francis Group, Milton Park, Abingdon-on-Thames, 231-255. 